International Journal of Distributed and Parallel Systems (IJDPS) Vol.3, No.5, September 2012

\title{
PerformanCe INVESTIgATION OF 802.11 MAC DCF
}

\author{
Siddharth Dutt Choubey ${ }^{1}$, Vikram Jain ${ }^{2}$, Rohit Singh Thakur ${ }^{3}$ \\ ${ }^{1,3}$ PG Department of CSE (SS \& CTA),SRIT, Jabalpur, INDIA \\ siddharth.choubey@gmail.com, rohit.singhthakur2@gmail.coom \\ ${ }^{2}$ Research Scholar, CMJ University, INDIA \\ vikram.sritegmail.com
}

\begin{abstract}
In the 802.11 protocol, DCF can be termed as an important mechanism in order to access the medium (Channel). This scheme is a random access based scheme which has its fundamentals based upon the efficient usage of CSMA/CA protocol. The retransmission of various collided packets is effectively managed in accordance with the Binary exponential Back-off rules. The waiting time of the BEB is exponentially increased by 2 after every unsuccessful transmission. Every successful transmission sets the back-off stage to initial stage and the contention window is also subsequently set to minimum regardless of any network conditions like the various $n$ number of competing nodes. As the number of competing nodes rises, it can cause substantial performance deprivation as a result of the new collisions caused. This paper highlights and investigates the various modifications possible in the basic calculating methodology of the $C W$ size after every successful transmission and collision of the BEB algorithm and it also evaluates the performance through different simulations possible for it. This paper also throws some significant light on the comparative study conducted on the throughput, end to end delay and packet loss ratio of the investigated schemes along with conventional DCF \& one-another.
\end{abstract}

\section{KEYWORDS}

802.11 MAC; DCF; CSMA/CD; BEB; WLAN; Contention window

\section{INTRODUCTION}

Military and commercial applications can be greatly benefited by efficiently using WLANs . In a WLAN, Transmission of packets takes palce in an unsynchronized fashion. Conflicts are minimize \& the shared channel is properly co-ordinate if MAC access control (MAC Layer) employs the protocol .There for the need for an effective mac protocol is adamant. In WLAN all connecting nodes are communicate via shared transmission channel (medium). The MAC layer provide, two mechanism (DCF \& PCF) for controlling the access of shared channel,.PCF is option mechanism but DCF is mandatory. Due to common transmission channel collision of packets is the very common in WLAN. The carrier sensing multiple access/collision avoidance (CSMA/CA) protocol and the binary exponential backoff (BEB) algorithm are two main component of DCF that are used to avoid collisions of packet [1][10].

\section{Operational Mode Of Conventional DCF}

In 802.11, DCF can be termed as a fundamental access method which is employed in order to facilitate asynchronous data transfer on best effort basis. It is already specified in the standards [1] that the DCF must be acceptable and enforceable to all the work stations within a Basic Service Set(BSS). DCF is primarily based upon CSMA/CA. In $802.11 \mathrm{CS}$ is performed at Physical Layer also called as Physical Carrier Sensing and MAC layer also termed as Virtual Carrier Sensing. [5][9].DCF allow medium sharing between nodes using CSMA/CA protocol. Two channel access mechanisms are used in DCF: Basic Access Mechanism \& RTS/CTS 
Mechanism. In basic access mechanism, on successful transmission, after a receiving of packet, the receiver node transmitted a positive MAC acknowledge (ACK) to sender. It is also known as two way handshaking mechanism. In RTS/CTS, before sending a packet, sender node tries to reserve the channel. if channel is idle, sender sends RTS frame first after receiving the RTS receiver send back CTS frame after the SIFS. After that actual packet is transmitted \& ACK response occurs. [1][8]. (for more details about DCF please use following reference $[1][2][3][5][8])$.

\subsection{Binary Exponential Back off Algorithm}

DCF utilizes the exponential back-off scheme. The back-off time for every packet transfer is chosen within the range of $0, \mathrm{~W}-1$ and in a uniform fashion. The value "w" is termed as Contention Window and it drastically depends upon the number of unsuccessful transmission for the chosen packet. For the very first transmission the value is set to CWmin also termed as minimum contention window and after every transmission that becomes unsuccessful the value of " $w$ " is doubled reaching to a maximum limit of :

$$
\mathbf{C W}_{\max }=2^{\mathrm{m}} * \mathbf{C} \mathbf{W}_{\min } .
$$

The back off time counter continually gets decremented until the channel is sensed in an "Idle" state. It goes in "Frozen" State when a transmission is detected on the channel and it goes to the reactivated state when the channel is sensed idle again for more than a DFIS. As soon as the back-off time reaches zero the station starts the transmission [1][2][3][8].

\subsection{Problems with Existing Architecture}

DCF is used in order to resolve collision through Contention Window and backoff time. As specified in the original standard [1], "after each successful transmission, the backoff stage will resume to the initial stage 0 , and the contention window will be set to $\mathbf{C W}_{\min }$ regardless of network conditions such as number of competing nodes". This method, referred to as "heavy decrease' tends to work well when the number of competing nodes is less. Substantial performance deprivation occurs when number of competing nodes rises \& causes new collision between the nodes.

The operation of existing DCF protocol can be summarized from the following figure -

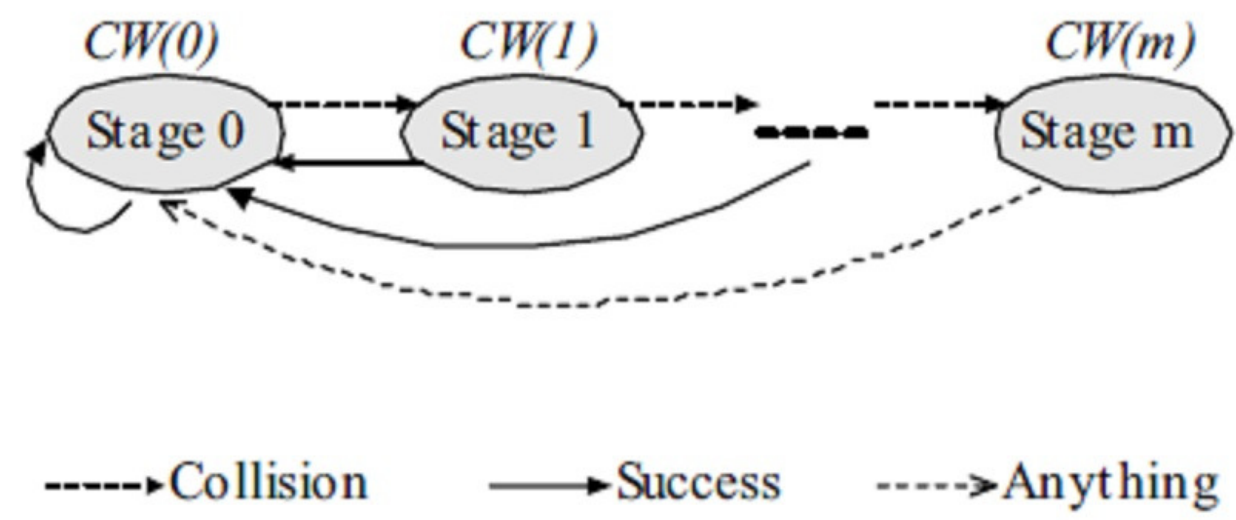

Figure 1.Operation of 802.11 DCF with BEB Algorithm

For example, let us assume that the current backoff stage is ' $\mathrm{i}$ ' with contention window 
$\boldsymbol{C W}$ ( i ) $=2^{\mathrm{i}} * \mathbf{C W}_{\min }$, and there is a successful transmission, the next backoff stage will be stage 0 with contention window $\boldsymbol{C W}(0)=31$ according to the specification. But if the number of competing nodes is large enough ( $>31)$, the new collision will likely occur at the backoff stage 0 . The main argument is that since the current backoff stage is ' $i$ ' some collision must have occurred recently at the previous stage. Now if the number of current competing nodes is larger than or close to $\boldsymbol{C W}$ ( i ), and if the backoff stage is set to 0 , there is a high probability that new collisions will happen. So resetting the contention window after every successful transmission is an inefficient approach if the number of nodes is large. The working of BEB algorithm can be summated as follows:

$$
\begin{aligned}
& \mathbf{C W}=\min \left[2^{*} \mathbf{C W}, \mathbf{C W}_{\max }\right], \text { upon collision } \\
& \mathbf{C W}=\mathbf{C W}_{\min }, \text { upon success }
\end{aligned}
$$

We also observe that fast build-up is caused when the waiting times uniformly spreads the backlog traffic subsequently over a larger time frame but in case of MANET this rapid build-up of the waiting time along with increasing number of various occurrences of collisions cannot be termed appropriate, wherein the contending nodes ultimately succumb to the geographic location of the contention and are displaced due to their mobility. Therefore the node must not be made to wait for the durations as the waiting times vary exponentially with a binary base [1][8][10].

\section{MODIFICATION IN BEB}

The IEEE 802.11 DCF employs BEB as a stability strategy to share the medium. But its contention window resetting mechanism degrades the performance of a network.(already described in section 2.2 ) In this section, We used five schemes to Modified the CW size after a transmission \& Collision to investigate the performance of the IEEE 802.11 DCF. On collision we change the default multiplication factor $\&$ on successful transmission we modify the default Resetting scheme of 802.11 to according to pseudo codes (Table1 \& Table 2) shown below. We investigate our simulation using following schemes.

Table 1. Schemes for resetting the CW on collision.

\section{Schemes for resetting the $\mathrm{CW}$ on collision}

Scheme 1

$\mathrm{CW}=\min \left[2 * \mathrm{CW}, \mathrm{CW}_{\max }\right]$ on a collision

Scheme 2

$\mathrm{CW}=\min \left[1.5 * \mathrm{CW}, \mathrm{CW}_{\max }\right]$ on a collision

Scheme 3

$\mathrm{CW}=\min \left[1.4 * \mathrm{CW}, \mathrm{CW}_{\max }\right]$ on a collision

Scheme 4

$\mathrm{CW}=\min \left[1.7 * \mathrm{CW}, \mathrm{CW}_{\max }\right]$ on a collision

Scheme 5

$\mathrm{CW}=\min \left[3 * \mathrm{CW}, \mathrm{CW}_{\max }\right]$ on a collision 
International Journal of Distributed and Parallel Systems (IJDPS) Vol.3, No.5, September 2012

Table 2. Pseudo code for Resetting CW since transmission was successful.

Pseudo code for Resetting CW since transmission was successful. (It will remain same for scheme 1 to 5)

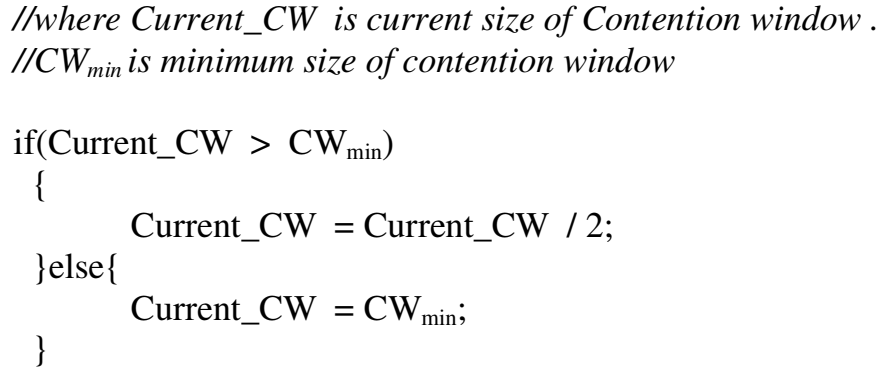

\section{SimULATION}

Design and implementation of schemes have been carried out using Global Mobile Information System Simulator (GloMoSim) which is a scalable simulation environment for large wireless and wired communication networks. The simulation under the study was a network that comprised of nodes that were placed in the $1500 \times 1500 \mathrm{~m} 2$ area. The data rate is $11 \mathrm{Mbps}$ and random waypoint mobility (RWMM) is applied to study the node movement. In RWMM, the nodes travel at a uniformly and evenly distributed speed [ MIN SPEED, MAX SPEED]. The simulation of every node is initiated by its movement towards a randomly chosen destination also known as waypoint. After the node reaches the waypoint it is made to rest for a PAUSE time. It then again selects a new waypoint and starts its movement towards it. This selection of new waypoint and movement towards it by the node is repeated until the simulation time is completed. In the simulation in this paper the pause time is set to 0 which means that the movement of the modes is continuous throughout the entire simulation. This is done in order to gain a proper insight about the worst case scenario regarding the impact of the node mobility.

\subsection{Simulation Parameter}

Table 3. Simulation Parameters

\begin{tabular}{|l|l|}
\hline Parameter & Value \\
\hline Mobility Model & Random Waypoint \\
\hline Speed of Mobile Node & $\begin{array}{l}\text { Uniformly distributed Between [0,10] } \\
\mathrm{m} / \mathrm{sec}\end{array}$ \\
\hline Propagation model & Two Ray \\
\hline Area (in $\mathrm{m}^{2}$ ) & $1500 \mathrm{x} \mathrm{1500}$ \\
\hline Channel Frequency & $2.4 \mathrm{GHz}$ \\
\hline Data Rate & $11 \mathrm{Mbps}$ \\
\hline MAC protocol & 802.11 DCF with backoff values \\
& $2,1.4,1.5,1.7,3$ (Table 1) on collision \& \\
& according to Pseudo code (Table 2) on \\
& Success. \\
\hline
\end{tabular}




\section{RESUlTS OBTAINED}

A series of simulations were performed and the new Scheme was tested against the conventional protocol for parameters such as Average end-to-end-delay, throughput and average packet loss ratio. The following graphs were plotted from the obtained output -

\subsection{Throughput Analysis}

Figures 5.1(a), 5.1(b), 5.1(c), 5.1(d), 5.1(e) shows the individual graph between No. of Nodes $\&$ Avg. Throughput of network on different scheme 1,2,3,4 \& 5 respectively. Figure 5.1(f) shows the graph between all schemes with conventional DCF \& it also shows the comparisons between schemes. Scheme 1,3,4 \& 5 gives better throughput ,when number of node in less (less than 35) as compare to conventional DCF. Scheme 1 also gives relatively fair throughput as compare to other schemes used in simulation. The simulation result also shows that scheme 1 is better when number of node is high (more than 60) in network.

\subsubsection{Scheme 1}

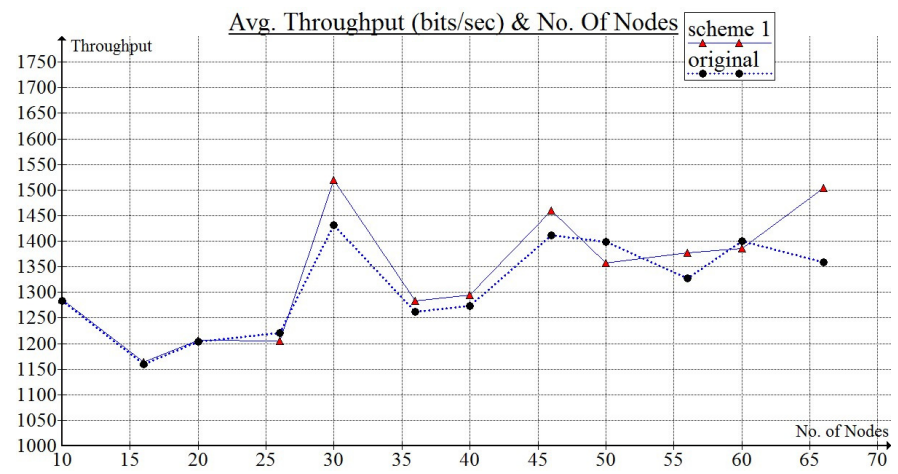

Figure 5.1(a) Throughput Vs Number of nodes

\subsubsection{Scheme 2}

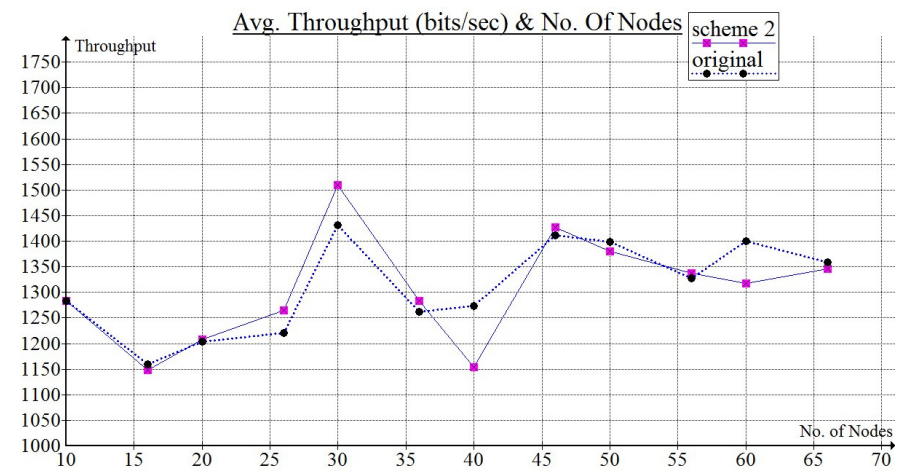

Figure 5.1(b) Throughput Vs Number of nodes 


\subsubsection{Scheme 3}

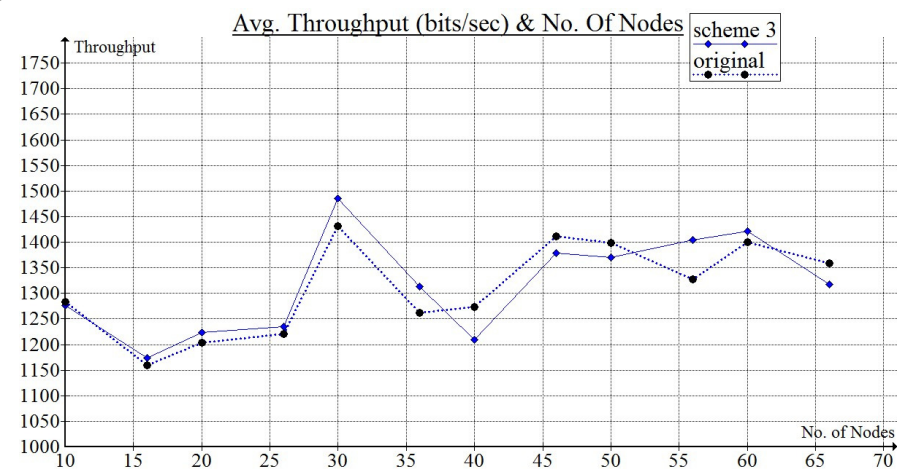

Figure 5.1(c) Throughput Vs Number of nodes

\subsubsection{Scheme 4}

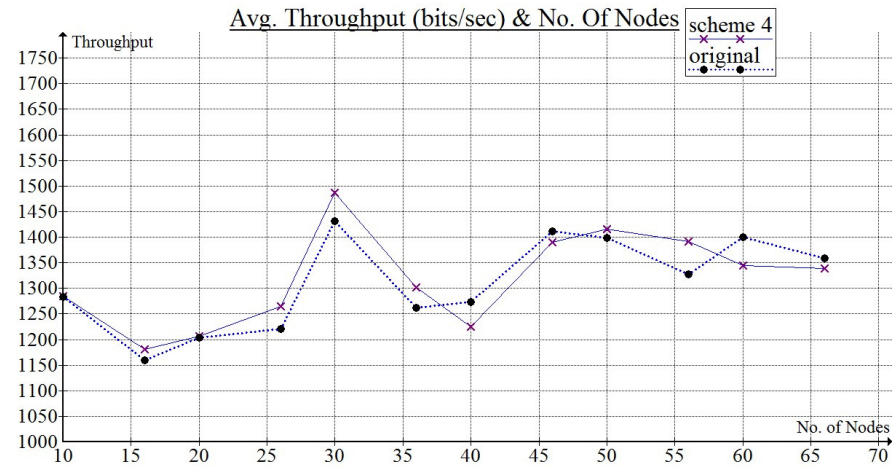

Figure 5.1(d) Throughput Vs Number of nodes

\subsubsection{Scheme 5}

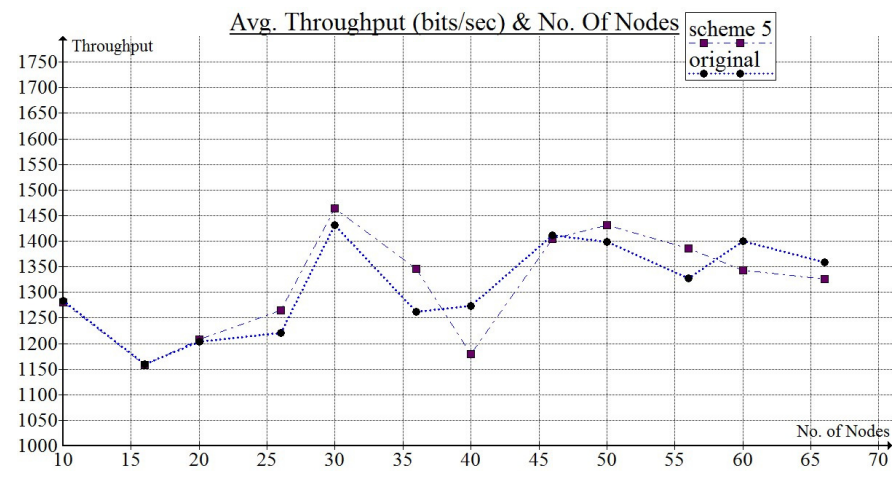

Figure 5.1(e) Throughput Vs Number of nodes 


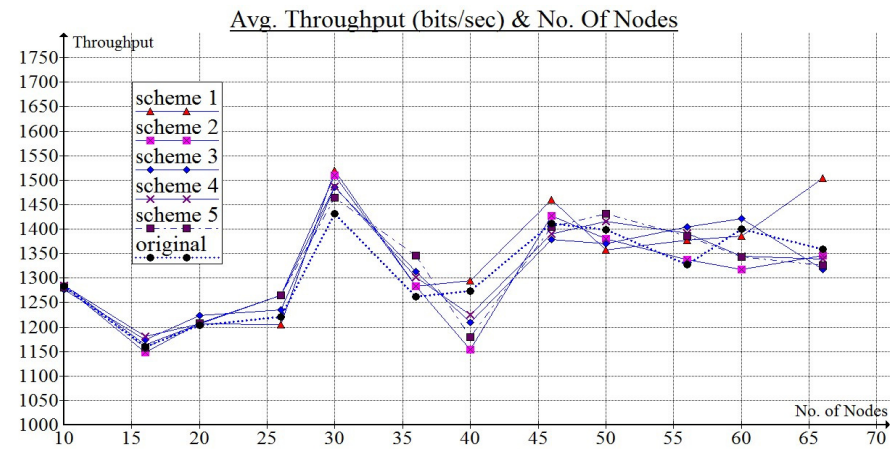

Figure 5.2(f) Comparison of Throughputs for all schemes

\subsection{Average End-to-End Delay}

Figures 5.2(a), 5.2(b), 5.2(c), 5.2(d) , 5.2(e) shows the individual graph for Average End-to-End Delay between Original DCF \& different scheme 1,2,3,4 \& 5 respectively. Figure 5.2(f) shows the graph between all schemes with original BEB \& it also shows the comparisons between schemes. The average end-to-end delay does not differ much for the different values of backoff factor. when the number of nodes in the network is few (less or equal to 16) scheme $2 \& 3$ gives better result as compare to conventional DCF. However, as the number of nodes increases in the network (more than or equal to 40), the average end-to-end delay decreases in scheme $2 \& 3$ as compared to conventional DCF protocol. But Scheme 3 gives the least average end-to-end delay as compared to conventional DCF \& other investigated schemes for high node density network.

\subsubsection{Scheme 1}

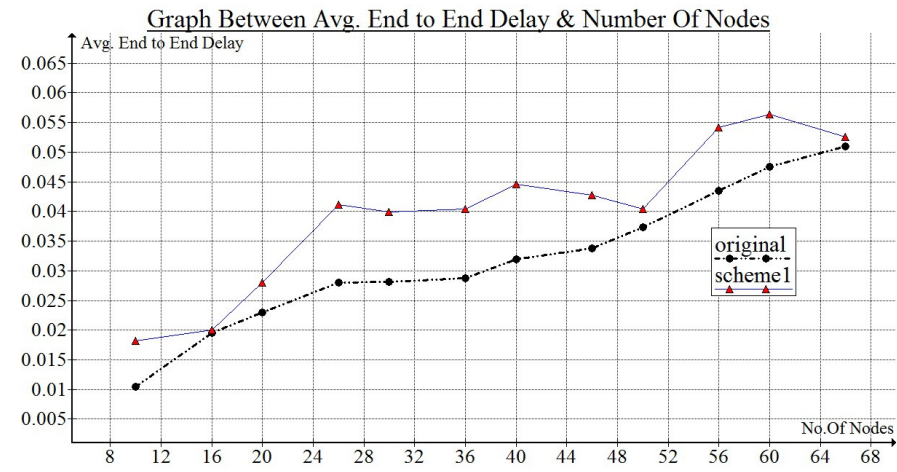

Figure 5.2 (a)Average end to end delay vs Number of nodes

\subsubsection{Scheme 2}

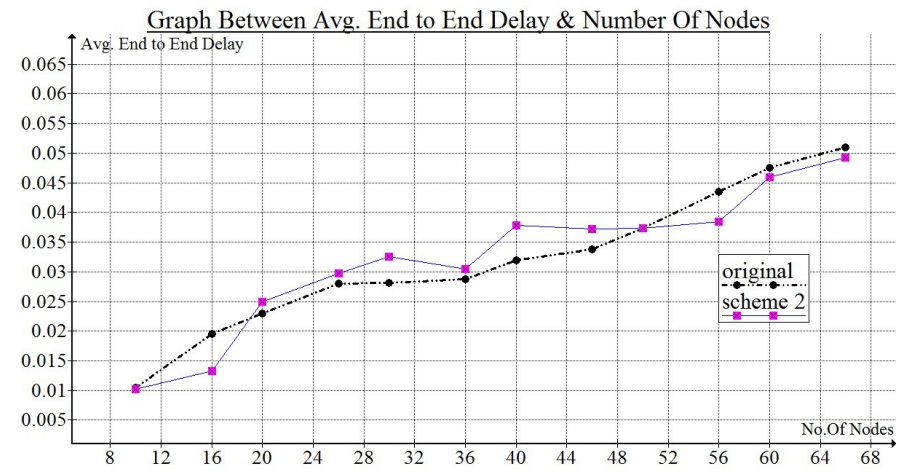

Figure 5.2 (b)Average end to end delay vs Number of nodes 
International Journal of Distributed and Parallel Systems (IJDPS) Vol.3, No.5, September 2012

\subsubsection{Scheme 3}

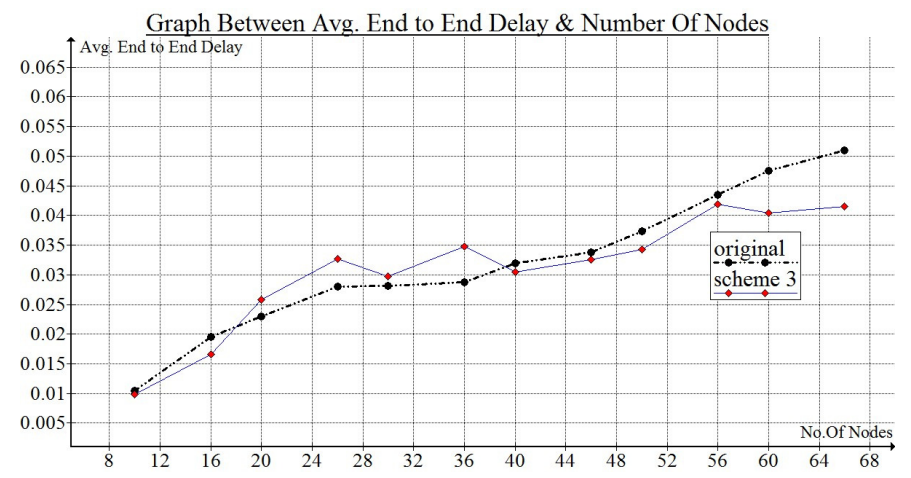

Figure 5.2 (c)Average end to end delay vs Number of nodes

\subsubsection{Scheme 4}

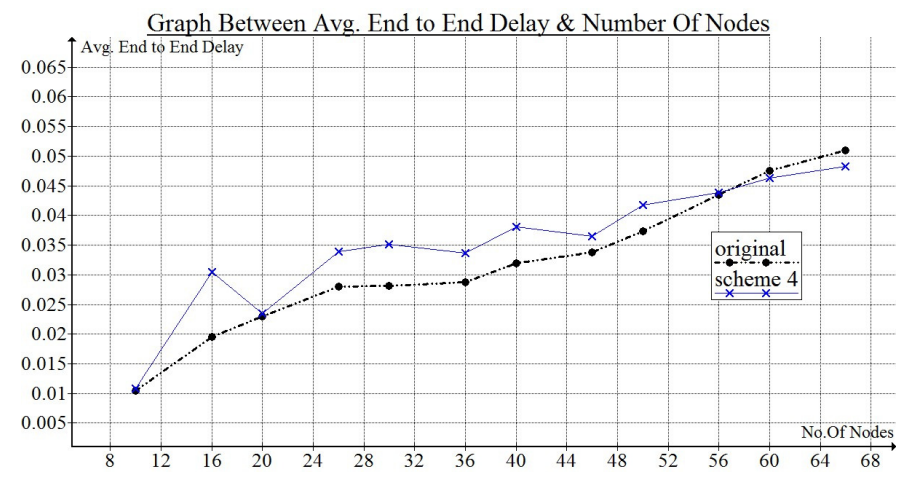

Figure 5.2 (d)Average end to end delay vs Number of nodes

\subsubsection{Scheme 5}

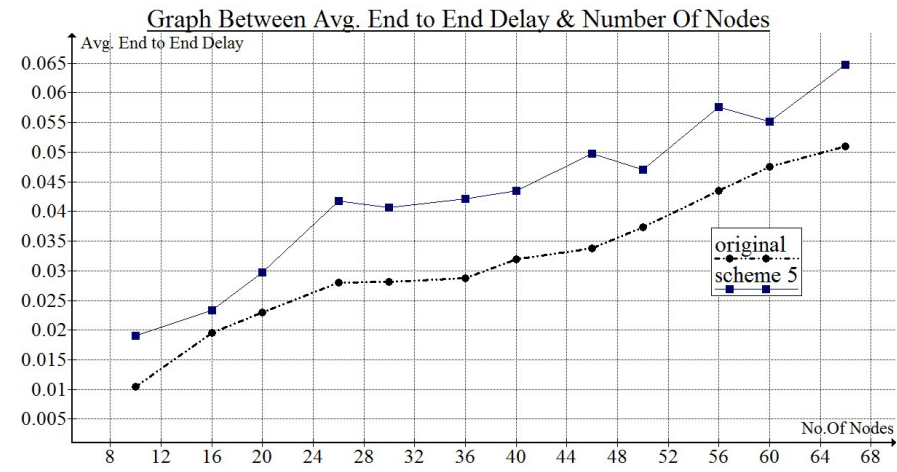

Figure 5.2 (e)Average end to end delay vs Number of nodes 


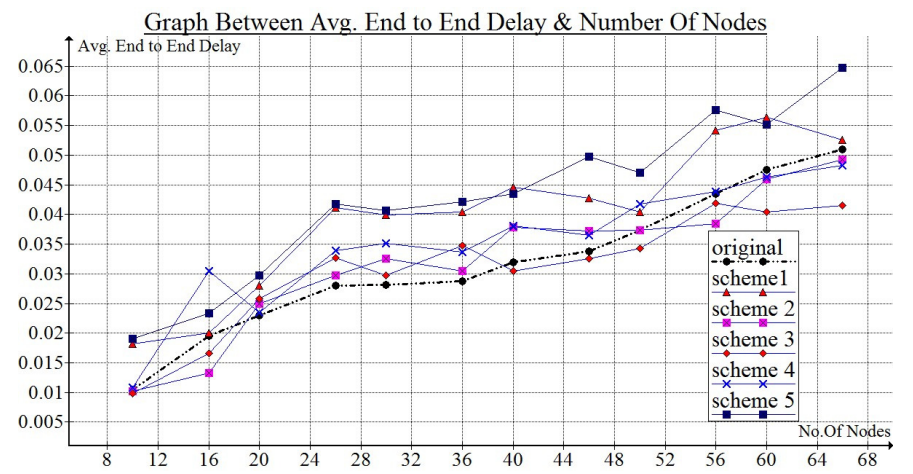

Figure 5.2(f) Comparison of Average end to end delay for all schemes

\subsection{Average packet loss ratio analysis}

Figures 5.3(a), 5.3(b), 5.3(c), 5.3(d) , 5.3(e) shows the individual graph for Average packet loss ratio analysis on different scheme 1,2,3,4 \& 5 respectively. Figure 5.3(f) shows the graph between all schemes with original BEB \& it also shows the comparisons between schemes. The graph in figure 5.3(f) suggests that as the number of nodes in the network increases $(=>60)$, conventional DCF's packet success rate decreases. But in small number of nodes $(<40)$ scheme $2,3 \& 4$ gives better result as compare to conventional DCF .In other words, conventional DCF has the maximum average packet loss ratio. Scheme $3,4 \& 5$ have the highest success rate in small number of nodes and hence the minimum packet loss ratio.

\subsubsection{Scheme 1}

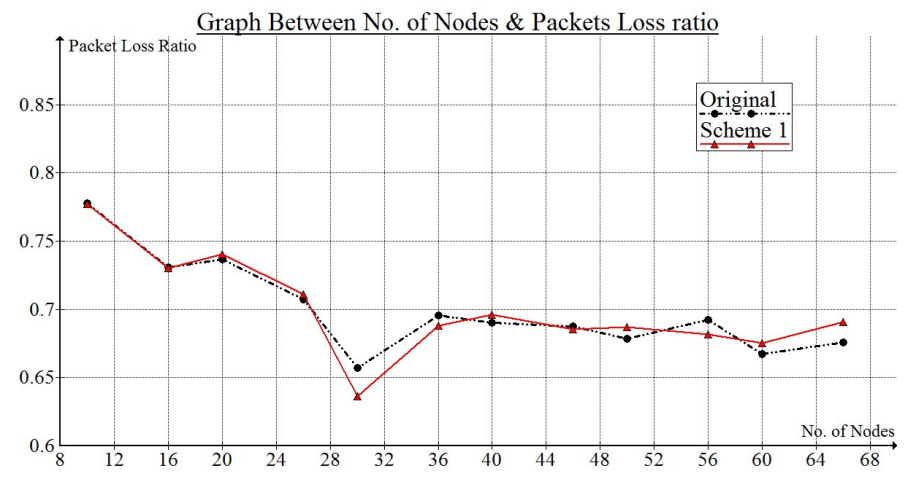

Figure 5.3(a) Packet Success Rate Vs Number of Nodes

\subsubsection{Scheme 2}

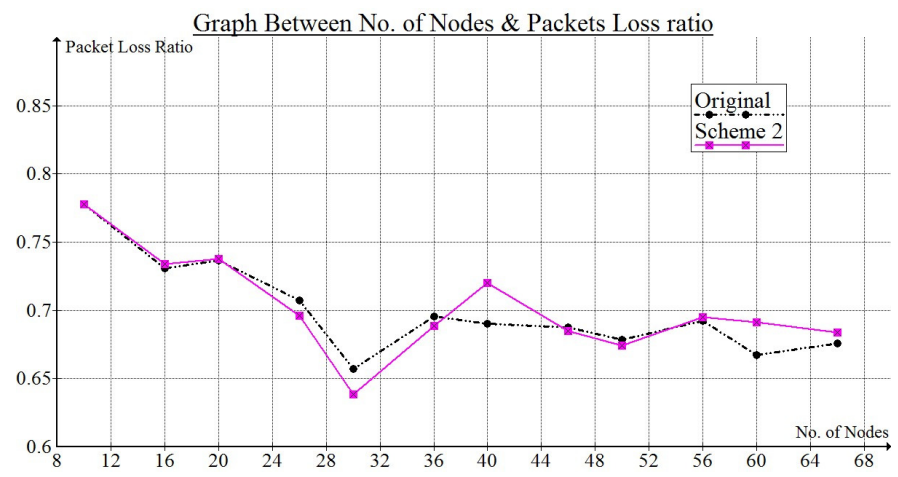

Figure 5.3(b) Packet Success Rate Vs Number of Nodes 
International Journal of Distributed and Parallel Systems (IJDPS) Vol.3, No.5, September 2012

\subsubsection{Scheme 3}

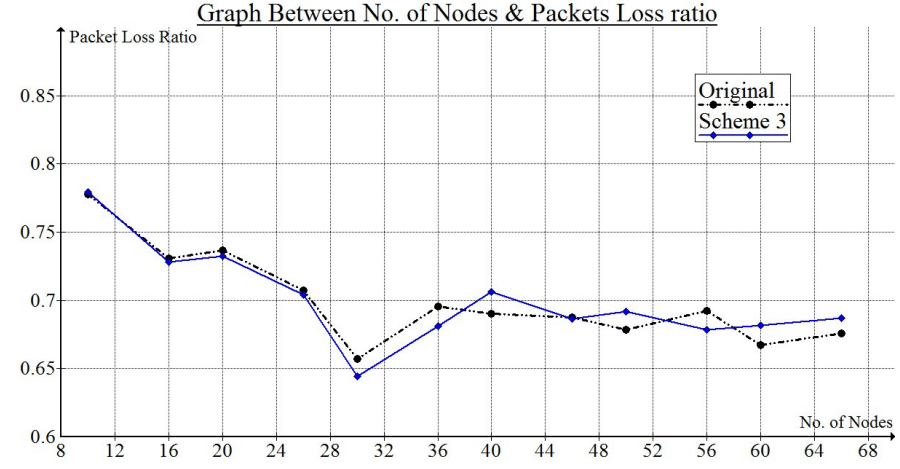

Figure 5.3(c) Packet Success Rate Vs Number of Nodes

\subsubsection{Scheme 4}

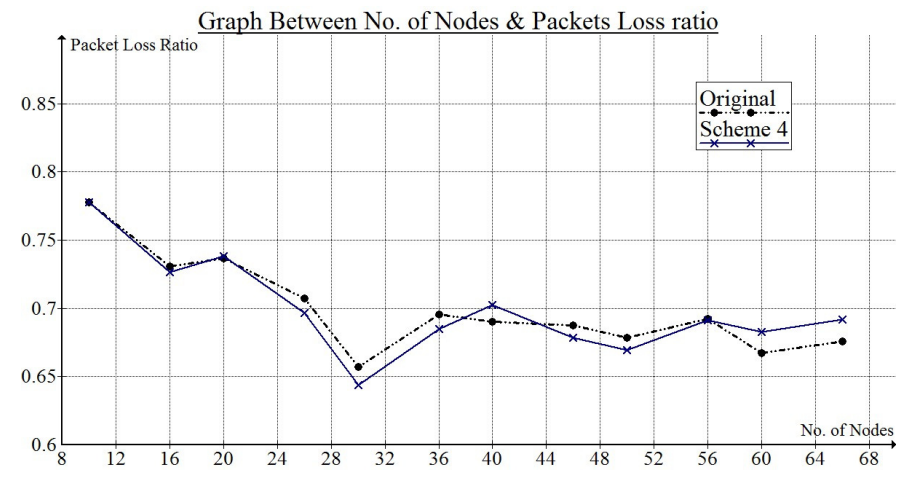

Figure 5.3(d) Packet Success Rate Vs Number of Nodes

\subsubsection{Scheme 5}

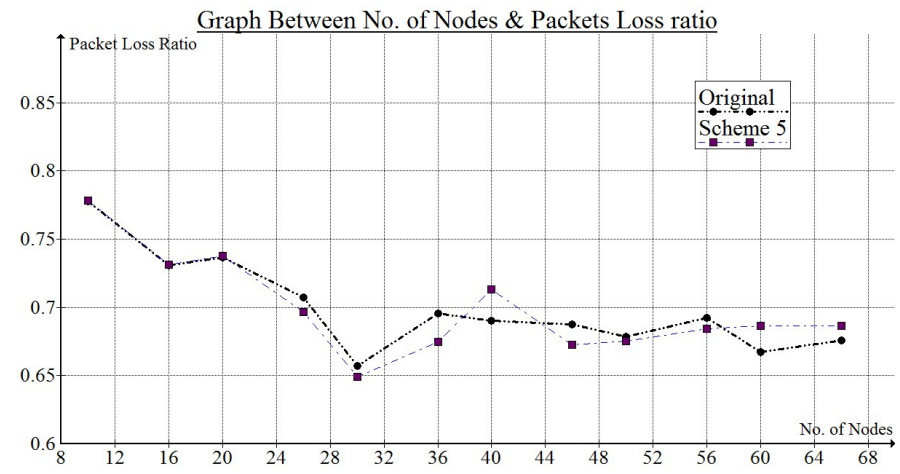

Figure 5.3(e) Packet Success Rate Vs Number of Nodes 


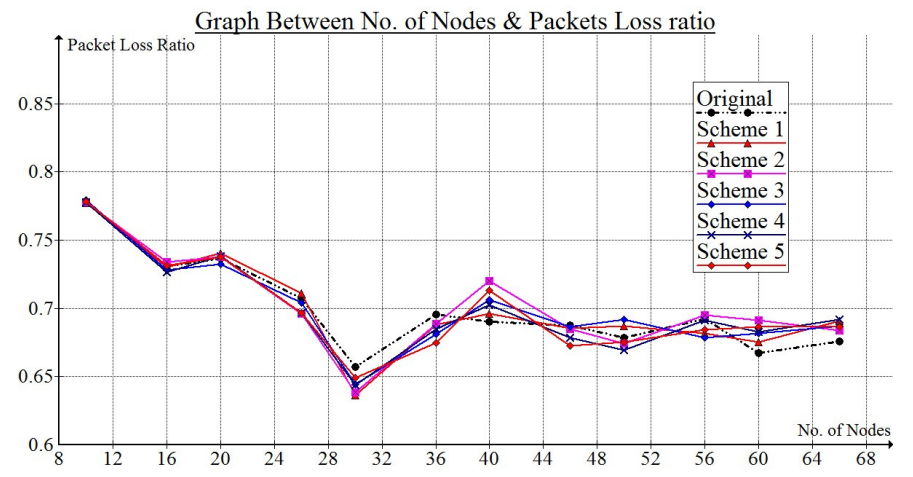

Figure 5.3(f) Comparison of Packet Loss Ratio for all schemes

\section{CONCLUSION \& FUTURE WORK}

In this paper, we have investigated new schemes for DCF protocol by modifications in the Binary Exponential Backoff algorithm. Different values for backoff factors were tested and compared against the conventional IEEE 802.11 DCF protocol. IEEE 802.11 has several disadvantages in that it's throughput decreases as the number of nodes in the network increases, average end to end delay is more, and there is a higher packet loss ratio in high node density networks. Simulation for the schemes were carried out using GloMoSim simulator and simulation results shows that - Scheme 1 gives better throughput for higher (more than or equal to 40 nodes) \& scheme 2 is better in small (less than or equal to 16 nodes) number of nodes than other schemes \& conventional DCF. Scheme 1 also has minimum end to end delay. It has lower end to end delay and could be deployed in delay sensitive applications. When we talking about success rate, scheme 2 gives better result in small network of nodes \& scheme 3,4\&5 gives better result in both (small \& large network of nodes). Due to drops fewer packets in MAC level and these schemes can easily be extended to support priority applications. Finally, schemes are very easy to deploy. It does not need to estimate number of competing nodes in the network and requires no change in the message structure and access procedures of DCF. But selection of right contention window size for performance improvement in IEEE802.11 in MANET is still a big challenge .Our future work will be to find the number of nodes \& switches the schemes automatically according to available active nodes in the network.

\section{REFERENCES}

[1] IEEE 802.11 standard, "Wireless LAN Medium Access Control (MAC) and Physical Layer (PHY) Specifications,” IEEE Std. June 1999.

[2] C. Rama Krishna, Saswat Chakrabarti, and Debasish Dutta, "A modified backoff algorithm for IEEE 802.11 DCF based MAC protocol in a Mobile Ad-Hoc Network”, TENCON 2004. 2004 IEEE Region 10 Conference, Volume B, 21-24 Nov. 2004 Page(s):664 - 667 Vol. 2

[3] Chonggang Wang, Weiwen Tang, Kazem Sohraby, Bo Li, “A simple mechanism on MAC layer to improve the performance of IEEE 802.11 DCF", Broadband Networks, 2004. BroadNets 2004. Proceedings of First International Conference on Broadband networks 2004, Page(s):365 - 374.

[4] Guanghong Wang, Yantai Shu, Liang Zhang, Oliver W.W. Yang, "Improving DCF in IEEE 802.11 Wireless LAN", IEEE CCECE 2003. CanadianConference on Electrical and Computer Engineering, 2003. Volume 2, 4-7 May 2003 Page(s):919 - 922.

[5] Haitao Wu, Shiduan Cheng, Yong Peng, Keping Long, Jian Ma "IEEE 802.11 Distributed Coordination Function(DCF): Analysis and Enhancement”, 2002 IEEE Page(s):605-609. 
International Journal of Distributed and Parallel Systems (IJDPS) Vol.3, No.5, September 2012

[6] Manshaei M.H., Cantieni G.R., Barakat C., Turletti T, "Performance analysis of the IEEE 802.11 MAC and physical layer protocol", Sixth IEEE International Symposium on a World of Wireless Mobile and Multimedia Networks, 2005. WoWMoM 2005. 13-16 June 2005 Page(s):88 - 97.

[7] Haitao Wu, Yong Peng, Keping Long, Shiduan Cheng, "A simple model of IEEE 802.11 wireless LAN”, International Conferences on Info-tech and Info-net, 2001. Proceedings. ICII 2001 - Beijing. 2001 Volume 2, 29 Oct.-1 Nov. 2001 Page(s):514 - 519

[8] Giuseppe Bianchi "Performance Analysis of the IEEE 802.11 Distributed Coordination Function" IEEE Journal on selected areas in communications, Vol.18 No.3 March 2000.

[10]. B.P. Crow, J.G. Kim, IEEE 802.11 Wireless Local Area Networks, IEEE Communications magazine, Sept. 1997

[9] B. P. Crow, I. Widjaja, J. G. Kim, and P. Sakai ,"Investigation of the IEEE 802.11 Medium Access Control (MAC) Sub layer Functions." Department of Electrical and Computer Engineering ,University of Arizona, IEEE 1997 Page(s):126-133.

[10] H. Wu, S. Cheng, Y. Peng, K. Long, and J. Ma, "IEEE 802.11 distributed coordination function (DCF): Analysis and enhancement,” Proc. Intl. Conf. Commun. pp. 605-609, 2002. 